\title{
Quantitative measurement of heat transfer fluctuation in a pipe flow around an orifice plate using high-speed infrared thermography
}

\author{
Naoki SHIIBARA*, Hajime NAKAMURA* and Shunsuke YAMADA* \\ * Department of Mechanical Engineering, National Defense Academy \\ 1-10-20 Hashirimizu, Yokosuka-shi, Kanagawa 239-8686, Japan \\ E-mail: nhajime@nda.ac.jp
}

Received 10 June 2015

\begin{abstract}
The aim of the present study is to demonstrate the quantitative measurement of the spatio-temporal fluctuation of the heat transfer in a water pipe flow around an orifice plate. In the present study, a technique using high-speed infrared thermography was used to measure the heat transfer. The measurement was first performed for a fully developed pipe flow without installing an orifice plate in order to verify the validity of the measurement technique. The spatio-temporal distribution of the heat transfer coefficient was evaluated based on the temperature fluctuation of a heated thin-foil measured using high-speed infrared thermography, and the results indicated that quantitative measurement was possible not only for time-averaged values, but also for fluctuating values. The technique was then used to measure the heat transfer to the flow around an orifice plate (the bore ratio was approximately 0.5 ). As a result, it was revealed that the heat transfer downstream of the orifice fluctuated violently, and the instantaneous structure of the heat transfer was remarkably finer than the streaky structure for the fully developed pipe flow. The time-averaged value of the heat transfer had a maximum at approximately two diameters downstream of the orifice, where the rms value of the fluctuation and its characteristic frequency also became much higher than those for the fully developed pipe flow.
\end{abstract}

Key words : Turbulent flow, Unsteady flow, Pipe flow, Forced convection, Heat transfer, Separation, Reattachment, Spatio-temporal measurement, Infrared thermography

\section{Introduction}

Fluctuations in heat transfer between a fluid and a solid may cause high-cycle thermal fatigue, which results from repeated thermal stress in a solid wall when the amplitude of thermal stress due to wall temperature fluctuation exceeds the fatigue limit of the material. This effect has often been referred to as "thermal striping" in the piping systems of power plants (The Japan Society of Mechanical Engineers, 2003). In addition, unsteady conjugate heat transfer, which is a coupling phenomenon of heat transfer between fluid and solid, leads to an erroneous prediction of the total amount of heat transfer across the wall due to nonlinear time dependence (Mathie and Markides, 2013). Such effects lead to a deterioration in the accuracy and reliability of the thermal design of equipment, such as heat exchangers and thermal insulators. They are, however, rarely considered in actual thermal design.

In order to address these problems, it is important to measure heat transfer fluctuation quantitatively. Generally, measurements of the local heat transfer fluctuation have been performed by means of point measurements such as thermocouples and/or heat flux sensors. For example, thermocouples were used to directly measure wall temperature fluctuation (Tanaka et al., 2012, Miyoshi et al., 2014) or near-wall fluid temperature (Kimura et al., 2010) in order to investigate high-cycle thermal fatigue. Also, heat flux sensors have sometimes been used to measure heat flux fluctuation. Examples include: at the flow reattachment region of a backward-facing step (Kawamura et al., 1988, 1994); on a cylinder surface in cross flow (Scholten and Murray, 1997); downstream of a rectangular cylinder in channel flow (Nakagawa et al., 1998); and in exhaust pipe flow (Sorin et al., 2008). However, it is difficult to measure 
spatial distribution by means of point measurements - even with numerous sensors - because the heat transfer varies in a highly complicated manner.

Measurement techniques using infrared thermography, on the other hand, offer a distinct advantage. With infrared thermography it is possible to measure spatial temperature distribution without contacting the measurement surface. Such an advantage has made it possible in recent years to investigate instantaneous heat transfer distribution and its fluctuating pattern on a heated thin foil caused by flow turbulence (Hetsroni and Rozenblit, 1994, and Oyakawa et al., 2000). However, a major problem of the measurements conducted in these studies were the attenuation of temperature fluctuation due to thermal inertia of the test surface. Also, lateral conduction through the test surface attenuated the amplitude of the spatial temperature distribution. In order to overcome these problems, Nakamura (2007) proposed to restore the quantitative heat transfer fluctuation by solving the inverse heat conduction equation inside the test surface. Also, Nakamura and Yamada (2013) evaluated quantitative heat transfer fluctuation to the turbulent air flow, which varies in time and space using this technique.

In this study we first applied this technique to verify the validity of the measurement in the fully developed region of the water pipe flow. Then, we used this technique to investigate the separated and reattached flow behind an orifice, where heat transfer fluctuates violently.

\section{Nomenclature}

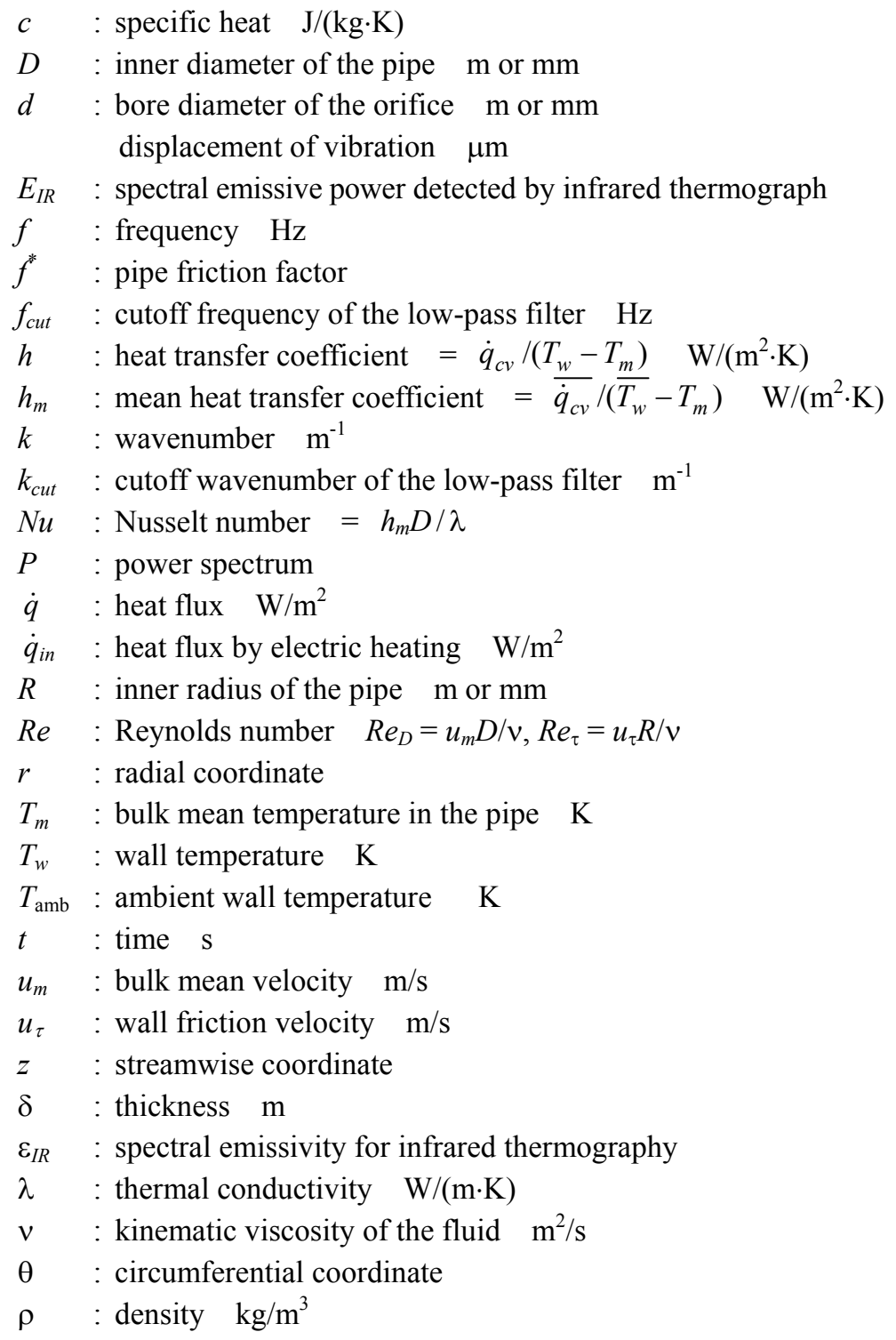




\section{Subscripts}

cd : conduction

$c v, c v r$ : convection to inside, convection to outside

$p, t \quad$ : black paint, titanium foil

$r d r \quad$ : radiation to outside

\section{Other Symbols}

( ),()$^{\prime}$ : mean value, fluctuation value

$\Delta($ ), ( ) rms: spatial or temporal amplitude, root-mean-square value

\section{Experimental apparatus}

The measurements were performed using the experimental setup shown in Fig. 1(a). This setup consists of (1) a head tank, (2) a rectifier tank, (3) an inlet region, (4) a test section, (5) an outlet region, and (6) an outlet tank. A header was placed on the rectifier tank in order to maintain the hydraulic head at $1,150 \mathrm{~mm}$. The inner diameter of the circular pipe was $D=20.4 \mathrm{~mm}$, and the length of the inlet region was $L=1,040 \mathrm{~mm}(L / D=51)$. A bell mouth was placed upstream of the inlet region, as shown in Fig. 1(b). Moreover, a turbulence promoter ( $\phi 1$ wire-shaped circular ring) was placed at the outlet of the bell mouth (inlet of the circular pipe). An orifice plate fabricated from acrylic resin was placed in the test section as shown in Fig. 1(c). The bore diameter was $d=10 \mathrm{~mm}$ (bore ratio: $d / D=0.49$ ). The thickness of the orifice plate was $2 \mathrm{~mm}$, and a clearance angle of $45^{\circ}$ was provided at the downstream edge. The mass flow rate of water, $Q$, was evaluated by measuring the weight of the water that overflowed from the inner outlet tank using a precision balance.

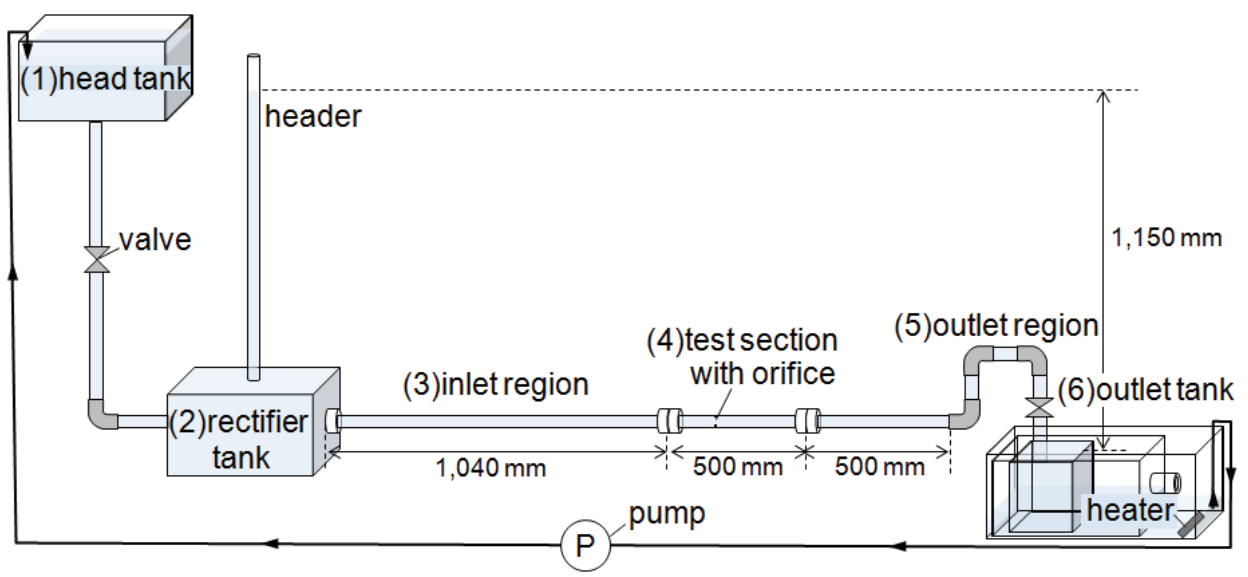

(a) Entire setup

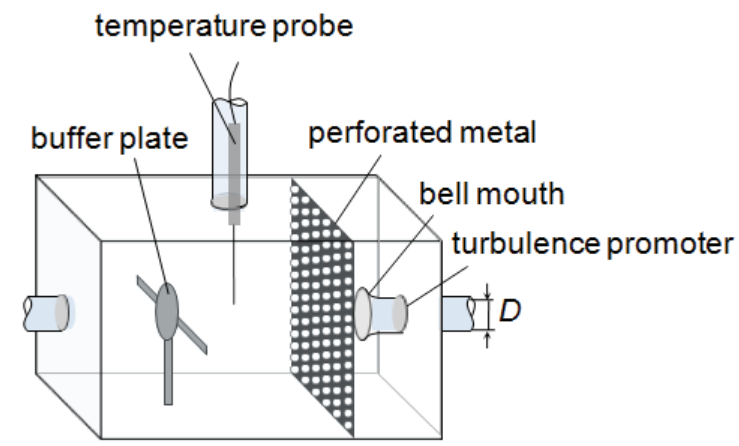

(b) Rectifier tank

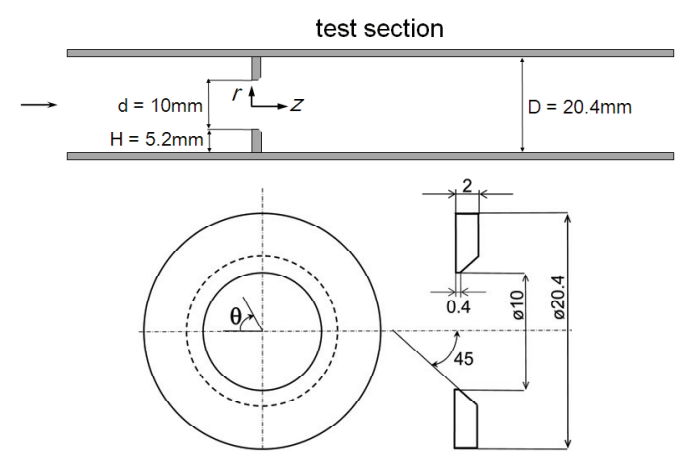

(c) Orifice plate

Fig. 1 Experimental setup. Water flow was generated by hydraulic head and was circulated by a water pump. 
The water was heated by a heater with a thermostat to approximately $30{ }^{\circ} \mathrm{C}$ and was circulated to the head tank using a water pump as shown in Fig. 1(a). The increase in the water temperature improves the accuracy of the infrared measurement, because the temperature difference between the heated surface at the test section $T_{w}$ and the ambient wall $T_{a m b}$ increases. The mean velocity in the pipe, $u_{m}=Q /\left(\rho \pi D^{2} / 4\right)$, was varied over 0.05 to $1.3 \mathrm{~m} / \mathrm{s}$. The Reynolds number, $R e_{D}$, based on the mean velocity was varied over 1100 to 38,700 .

Figure 2 shows the test section used for heat transfer measurement. Figures 2(a), 2(b), and 2(c) show a photograph, a top cross-sectional view, and schematic diagram, respectively, of the measurement system. The circular duct of the test section was fabricated from an acrylic pipe of $280 \mathrm{~mm}$ in length, the middle section of which was cut out in a semicircular shape over a length of $240 \mathrm{~mm}$. On the inner surface of the pipe, $20.7-\mu \mathrm{m}$-thick titanium foil was glued around the entire circumference, including the removed section. The orifice plate was placed into the test section as shown in Fig. 2(b). The upstream edge of the orifice plate was located $80 \mathrm{~mm}$ from the starting point of heating. In order to increase the emissivity of the heated surface, the outer surface of the titanium foil was coated with black paint (thickness: $\delta_{p} \approx 20 \mu \mathrm{m}$ ). The titanium foil was heated electrically through electrodes attached to both ends of the test section.

Although the titanium foil was bonded to the electrodes using an adhesive with high electrical conductivity, the contact resistance was not negligible. Therefore, the heat flux provided by electric heating was calculated based on the electrical resistivity of the titanium foil and the applied current, considering the dependence of temperature. The electrical resistivity was evaluated experimentally by measuring the voltage of a strip of the foil by applying a micro-current. The measured value was $5.58 \times 10^{-7} \Omega \mathrm{m}\left(20{ }^{\circ} \mathrm{C}\right)$, which was approximately the same as that of bulk titanium (The Japan Society of Thermophysical Properties, 2008).

Since the heat capacity of the heated surface (titanium foil coated with black paint) was very low along the removed section of the acrylic pipe, the wall temperature fluctuated according to the turbulent heat transfer to the water flow. The instantaneous temperature distribution and its temporal fluctuation on the test surface were measured using a high-speed infrared thermograph (SC4000, FLIR; IRT hereinafter), which can obtain thermo-images of 420 frames per second with a full resolution of $320 \times 256$ pixels. In this experiment, the frame rate increased up to $800 \mathrm{~Hz}$ with a reduction of pixels to $320 \times 128$. In order to suppress the diffuse reflection from the surroundings and to make the ambient wall temperature $T_{a m b}$ uniform, the test section was shielded by copper plates coated with black paint, as shown in Fig. 2(c).

The spectral emissivity of the test surface, $\varepsilon_{I R}$, was estimated based on the equivalent surface $(20.7-\mu \mathrm{m}$-thick titanium foil coated with 20 - $\mu$ m-thick black paint) fastened tightly to the heated copper plate. The spectral emissivity of

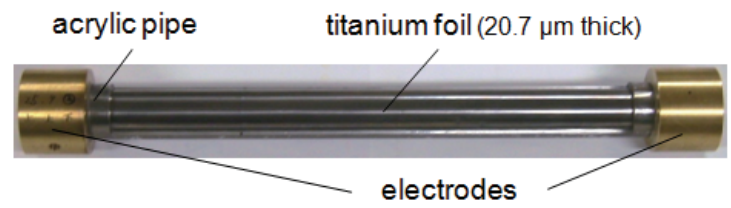

(a) Photograph

(before coating with black paint)

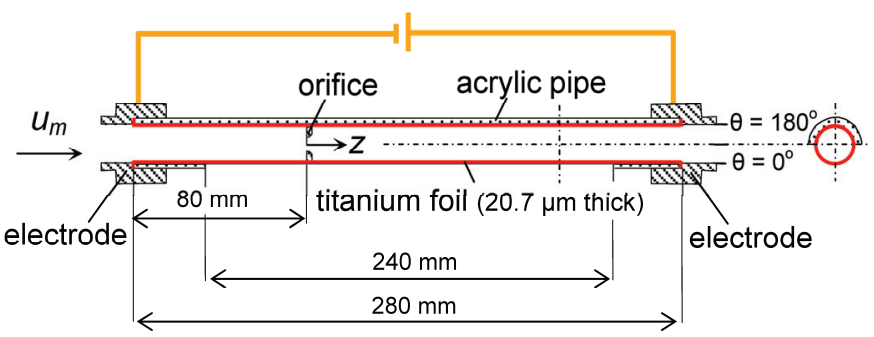

(b) Top cross-sectional view (with an orifice)

Fig. 2 Test section for heat transfer measurement. Test surface was composed of thin titanium foil coated with black paint and was heated electrically. The instantaneous temperature distribution and its temporal fluctuation on the test surface were measured using a high-speed infrared thermograph (SC4000, FLIR). 
the test surface can be calculated from the following equation, which expresses the spectral emissive power, $E_{I R}$, detected by the infrared thermograph:

$$
E_{I R}=\varepsilon_{I R} f\left(T_{w}\right)+\left(1-\varepsilon_{I R}\right) f\left(T_{a m b}\right),
$$

where $f(T)$ is the calibration function of the infrared thermograph for a blackbody, $T_{w}$ is the copper plate temperature $(\approx$ test surface temperature), and $T_{a m b}$ is the ambient wall temperature, all of which were measured using calibrated thermocouples. The terms of the right-hand side of Eq. (1) represent the emissive power from the test surface and the surroundings, respectively. The estimated emissivity, $\varepsilon_{I R}$, of the test surface used in this test was 0.90 .

\section{Experiment - Heat transfer in a fully developed pipe flow 3.1 Measurement of temperature distribution}

First, the measurement was performed in the fully developed region in a pipe flow without installing an orifice plate in order to verify the validity of the measurement technique using IRT.

Figure 3 shows instantaneous thermo-images of the heated surface measured using IRT downstream of the test section at $z / D=9.6$ to $13(z=193-260 \mathrm{~mm})$, where $z$ is the streamwise distance from the starting position of heating. Here, the circumferential axis, $\theta$, is not linearized, because the thermo-image of the pipe surface was measured as shown in Fig. 2(c). The average temperature difference between the heated surface and the water flow $\overline{T_{w}}-T_{m}$ was approximately $10{ }^{\circ} \mathrm{C}$ for each condition. For the laminar flow (Fig. 3(a), $R e_{D}=1,200$ ), the temperature in the upper region of the pipe increases due to the buoyancy effect, whereas the temperature distribution is completely steady.

On the other hand, for the turbulent flow (Fig. 3(b), $R e_{D}=11,200$ ), the temperature fluctuates due to the flow turbulence. In this case, the buoyancy effect was small because the time-averaged temperature is approximately uniform in the vertical direction. The temperature distribution for the turbulent flow is characterized by a streaky structure elongated along the streamwise direction, which flows downstream with some meandering.

\subsection{Calculation of heat transfer coefficient}

Figure 4 shows a schematic diagram of the heat transfer from the heated surface. If the temperature of the heated surface, which is composed of thin titanium foil coated with black paint, is assumed to be uniform in the thickness direction, the heat conduction equation for the heated surface is expressed as follows:

$$
\left(c_{t} \rho_{t} \delta_{t}+c_{p} \rho_{p} \delta_{p}\right) \frac{\partial T_{w}}{\partial t}=\lambda_{e}\left(\delta_{t}+\delta_{p}\right)\left(\frac{\partial^{2} T_{w}}{\partial z^{2}}+\frac{\partial^{2} T_{w}}{\partial(R \theta)^{2}}\right)+\dot{q}_{i n}-\dot{q}_{c v}-\dot{q}_{c v r}-\dot{q}_{r d r}
$$

where $T_{w}$ is local and instantaneous temperature of the heated surface measured using IRT, $c, \rho, \delta$, and $\lambda$ are the specific heat, density, thickness, and thermal conductivity, respectively. The subscripts $t$ and $p$ indicate the titanium foil and the black paint, respectively. Here, $\lambda_{e}=\left(\lambda_{t} \delta_{t}+\lambda_{p} \delta_{p}\right) /\left(\delta_{t}+\delta_{p}\right)$ is the effective thermal conductivity through the thin heated surface. Moreover, $\dot{q}_{i n}$ is the input heat flux to the thin foil due to Joule heating, and $\dot{q}_{c v}$ is the convective heat flux to the water flow, which is expressed as

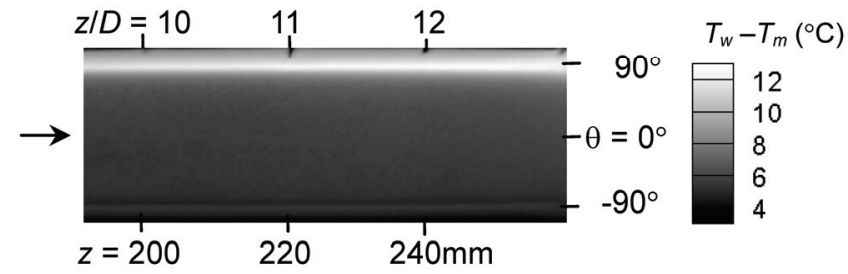

(a) $R e_{D}=1,200$ (laminar)

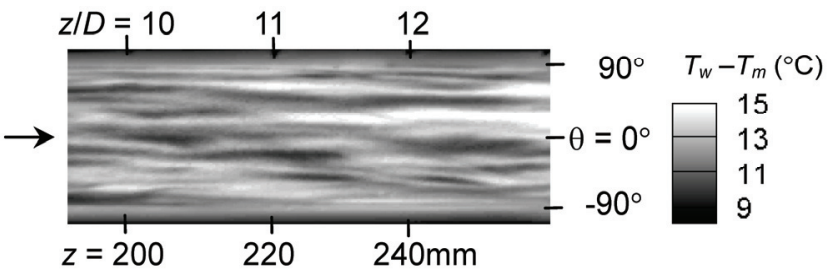

(b) $R e_{D}=11,200$ (turbulent)

Fig. 3 Thermo-images of the heated surface measured using IRT. 


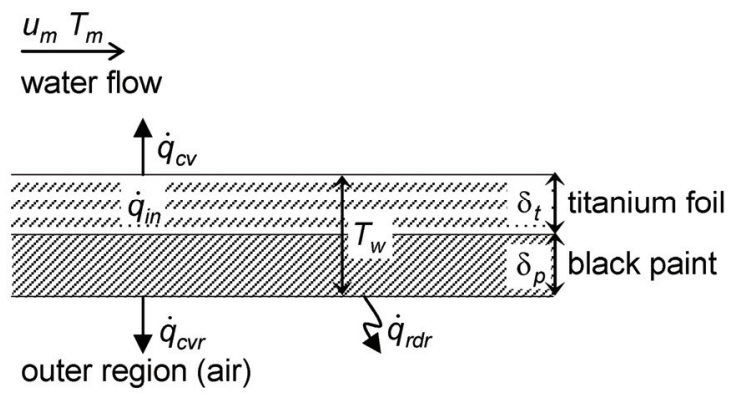

Fig. 4 Schematic diagram of the heat transfer from a heated surface, which is composed of thin titanium foil coated with black paint. $\dot{q}_{i n}$ is the input heat flux to the thin foil due to Joule heating. $\dot{q}_{c v}$ is the convective heat flux to the water flow. $\dot{q}_{c v r}$ and $\dot{q}_{r d r}$ are the heat fluxes to the outside due to convection and radiation, respectively.

$\dot{q}_{c v}=h\left(T_{w}-T_{m}\right)$,

and $\dot{q}_{c v r}$ and $\dot{q}_{r d r}$ are the heat fluxes to the outside due to convection and radiation, respectively. The local bulk temperature of the water flow, $T_{m}$, was calculated considering the temperature increase due to the convective heat flux to the water flow. Moreover, $\dot{q}_{c v r}$ was estimated using the empirical equation of natural convection around a horizontal circular cylinder (Fujii et al., 1982), and $\dot{q}_{r d r}$ was estimated from the emissive power based on the gray surface. (The total emissivity was assumed to be given by $\varepsilon_{I R}$.) The values of $\dot{q}_{c v r}$ and $\dot{q}_{r d r}$ were negligible compared to the input heat flux $\dot{q}_{i n} .\left(\dot{q}_{c v r}+\dot{q}_{r d r}\right.$ was less than $1 \%$ of $\left.\dot{q}_{i n}.\right)$

The local and instantaneous heat transfer coefficient to the water flow, which is derived from Eqs. (2) and (3), is expressed as follows:

$$
h=\frac{\dot{q}_{i n}-\dot{q}_{c v r}-\dot{q}_{r d r}+\lambda_{e}\left(\delta_{t}+\delta_{p}\right)\left(\frac{\partial^{2} T_{w}}{\partial z^{2}}+\frac{\partial^{2} T_{w}}{\partial(R \theta)^{2}}\right)-\left(c_{t} \rho_{t} \delta_{t}+c_{p} \rho_{p} \delta_{p}\right) \frac{\partial T_{w}}{\partial t}}{T_{w}-T_{m}}
$$

where $\lambda_{e}\left(\delta_{t}+\delta_{p}\right)\left(\partial^{2} T_{w} / \partial z^{2}+\partial^{2} T_{w} / \partial(R \theta)^{2}\right)$ is the lateral heat conduction through the thin heated surface, and $\left(c_{t} \rho_{t} \delta_{t}+c_{p} \rho_{p} \delta_{p}\right) \partial T_{w} / \partial t$ is the thermal inertia due to the heat capacity of the heated surface. Spatio-temporal attenuation of the temperature due to the lateral conduction and the thermal inertia can be restored by calculating these derivative terms. In the calculation of the present data, the time differential $\Delta t$ in Eq. (4) was set to the frame interval of the thermo-images ( $\Delta t=1.25-10 \mathrm{~ms}$, depending on $R e_{D}$ ), and the space differential, $\Delta z$, and $\Delta(R \theta)$ were set to the pixel pitch of the thermo-images $(\Delta z \approx \Delta(R \theta) \approx 0.2 \mathrm{~mm})$.

The fine-scale noise in the spatio-temporal distribution significantly affects the determination of the time derivative term $\Delta T_{w} / \Delta t$ and space derivative terms $\Delta^{2} T_{w} / \Delta z^{2}$ and $\Delta^{2} T_{w} / \Delta(R \theta)^{2}$. Thus, it is essential to reduce the fine-scale noise in calculating these derivative terms. In order to address this concern, low-pass filtering was applied to the temporal $(t)$ and spatial $(z$ and $\theta$ ) directions separately. The filter used here eliminates all frequencies (wavenumbers for the $z$ and $\theta$ directions) above the cutoff frequency $f_{\text {cut }}$ (cutoff wavenumber $k_{\text {cut }}$ ), whereas frequencies below the $f_{\text {cut }}\left(k_{\text {cut }}\right)$ pass though the filter unchanged. The values of $f_{c u t}$ and $k_{c u t}$ can be adjusted separately to reduce the noise effectively while not attenuating the structure caused by flow turbulence (Nakamura and Yamada, 2013).

Incidentally, the assumption of uniform temperature across the thickness of the heated surface does not hold if the fluctuation frequency increases significantly. In fact, the restoration rate, which is the ratio of the heat transfer amplitude obtained using Eq. (4) to the original value, decreases with increasing frequency. In order to examine the effect of this feature, a simple one-dimensional heat conduction analysis (along the thickness direction) was conducted for the condition of a fully developed pipe flow at $R e_{D}=12,100$ (Shiibara et al., 2013). As a result, the restoration rate 
was approximately unity at $f<10 \mathrm{~Hz}$. In contrast, the restoration rate gradually decreased with increasing frequency at $f$ $>10 \mathrm{~Hz}$, and became approximately $90 \%$ at $f=80 \mathrm{~Hz}$. Under the present experimental condition, the attenuation increased with the Reynolds number at $R e_{D} \geq 15,000$ for the fully developed pipe flow, because a part of the fluctuation frequency exceeded $80 \mathrm{~Hz}$ (Shiibara et al., 2013).

\subsection{Verification of the time-averaged value}

Figure 5 shows the Nusselt number measured at $z / D=10.8(z=220 \mathrm{~mm})$ with respect to the Reynolds number, $R e_{D}$. Here, the Nusselt number was calculated using the mean heat transfer coefficient, $h_{m}$, which was defined by the time-averaged wall temperature, $\overline{T_{w}}$, as follows:

$$
N u=\frac{h_{m} D}{\lambda}=\frac{\overline{\dot{q}_{c v}}}{\overline{T_{w}}-T_{m}} \frac{D}{\lambda}
$$

In the developing region of the temperature field, the local Nusselt number can be expressed by the following empirical equation (Gnielinski, 1976):

$$
N u(z)=N u_{\infty}\{1+C /(z / D)\}
$$

At the position of $z / D=10.8(C=0.2$ at $\operatorname{Pr} \approx 5)$, the ratio of the Nusselt number to the fully developed Nusselt number, $N u / N u_{\infty}$, is 1.02 . This indicates that the temperature field at this position is almost fully developed.

The Nusselt number obtained here is compared with the Nusselt number obtained by empirical equations, such as that of Petukhov (1970), which was developed based on an analogy between the heat and the momentum transport:

$$
N u_{\infty}=\frac{\left(f^{*} / 2\right) \operatorname{Re} e_{D} \operatorname{Pr}}{1.07+12.7 \sqrt{f^{*} / 2}\left(\operatorname{Pr}^{2 / 3}-1\right)} \quad\left(10^{4}<\operatorname{Re}_{D}<10^{6}, 0.5<\operatorname{Pr}<2000\right),
$$

where $f^{*}$ is the pipe friction factor derived from the Blasius formula. We also compared the Nusselt number obtained

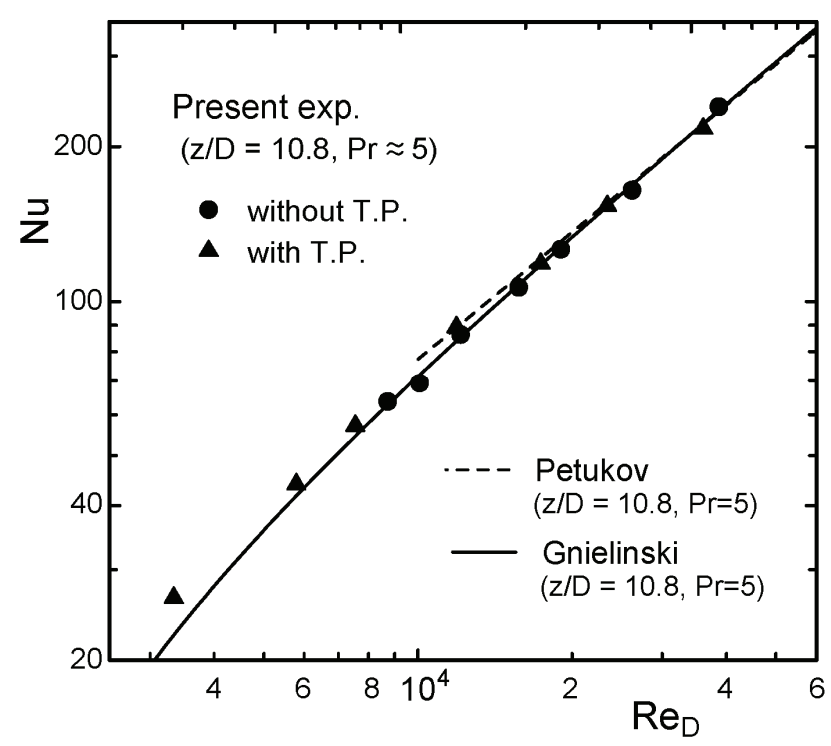

Fig. 5 Nusselt number with respect to the Reynolds number. The present experimental data agree well, within $5 \%$, of the empirical equation of Gnielinski for $R e_{D}>5,000$. 
herein with that obtained by the empirical equation of Gnielinski (1976), which was extended to the low-Reynolds-number region:

$$
N u_{\infty}=\frac{\left(f^{*} / 2\right)\left(\operatorname{Re}_{D}-1000\right) \operatorname{Pr}}{1+12.7 \sqrt{f^{*} / 2}\left(\operatorname{Pr}^{2 / 3}-1\right)} \quad\left(2300<\operatorname{Re}_{D}<10^{6}, 0.5<\operatorname{Pr}<2000\right) .
$$

These empirical equations (Eqs. (7) and (8)) are plotted in Fig. 5. The present experimental data agree well, within $5 \%$, of the empirical equation of Gnielinski for $R e_{D}>5,000$. This indicates that quantitative measurement is possible using the present measurement technique for the time-averaged value. In another aspect, the turbulence promoter (T.P.) placed at the inlet of the pipe had little effect on the heat transfer coefficient at the test section of the pipe (Fig. 1(b)).

\subsection{Verification of the fluctuating value}

Figure 6 shows the spatio-temporal distribution of the heat transfer coefficient at $\operatorname{Re}_{D}=10,100$ : (a) is the instantaneous distribution and (b) is the time trace of the circumferential distribution at $z / D=10.8(z=220 \mathrm{~mm})$. Here, the circumferential axis, $\theta$, was transformed to be linear. In the instantaneous distribution of Fig. 6(a), a streaky structure, corresponding to the thermo image of Fig. 3(b), appears, which elongates along the streamwise direction, similar to the instantaneous distribution for a turbulent boundary layer on a flat plate (Nakamura and Yamada, 2013). Note that the local heat-transfer frequently increases rapidly for a short duration (20-50 ms in this case), as shown in Fig. 6(b). This is caused by the convection of the high heat transfer spot in the streaky structure in Fig. 6(a).

To our knowledge, there have been no experimental studies on the spatio-temporal distribution of the turbulent heat transfer in a circular pipe. Moreover, very few numerical studies have been performed under conditions equivalent to the present experiment. Thus, it is difficult to validate the spatio-temporal structure obtained herein based on previous studies. However, it is possible to make a qualitative comparison with, for example, the instantaneous temperature distribution of direct numerical simulations (DNS hereinafter) reported by Saad et al. (2007) performed at $R e_{D}=5,500$ and $\operatorname{Pr}=0.71$ under the isoflux condition. The streaky structure in their study is similar in a visual sense to that

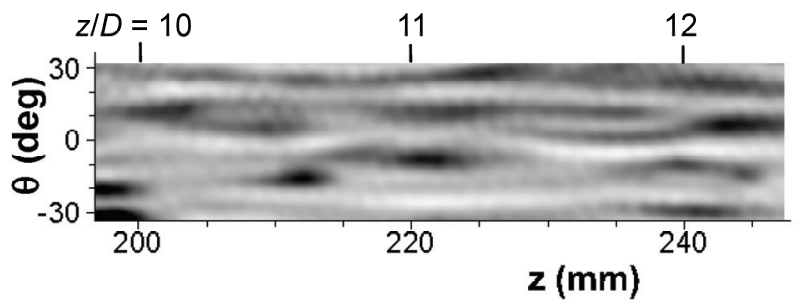

(a) Instantaneous distribution

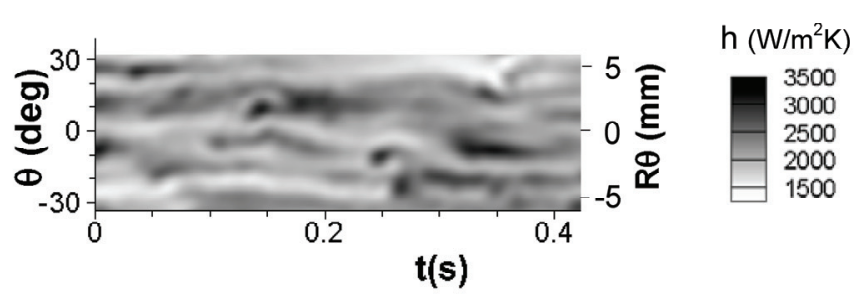

(b) Time trace along $\theta$ at $z / D=10.8(z=220 \mathrm{~mm})$

Fig. 6 Spatio-temporal distribution of heat transfer coefficient in a fully developed pipe flow, which is calculated by Eq. (4) $\left(R e_{D}=10,100, k_{\text {cut }}=1,190 \mathrm{~m}^{-1}, f_{\text {cut }}=60 \mathrm{~Hz}\right)$.

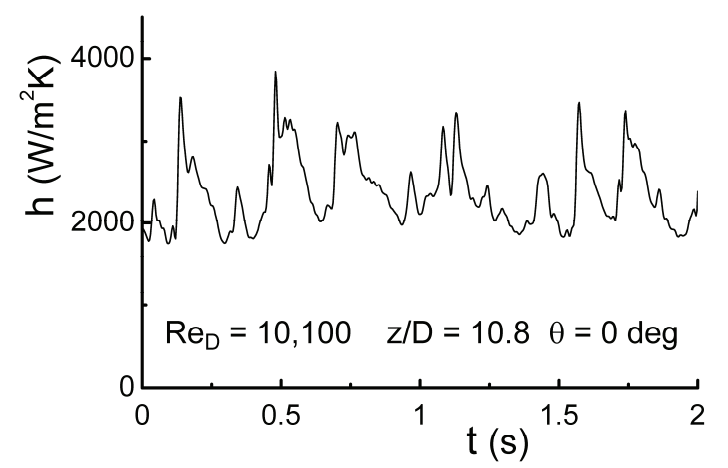

Fig. 7 Temporal fluctuation of the heat transfer coefficient, which is corresponding to Fig.6 $\left(\operatorname{Re}_{D}=10,100, z / D=\right.$ 10.8, $\theta=0^{\circ}$ ). 


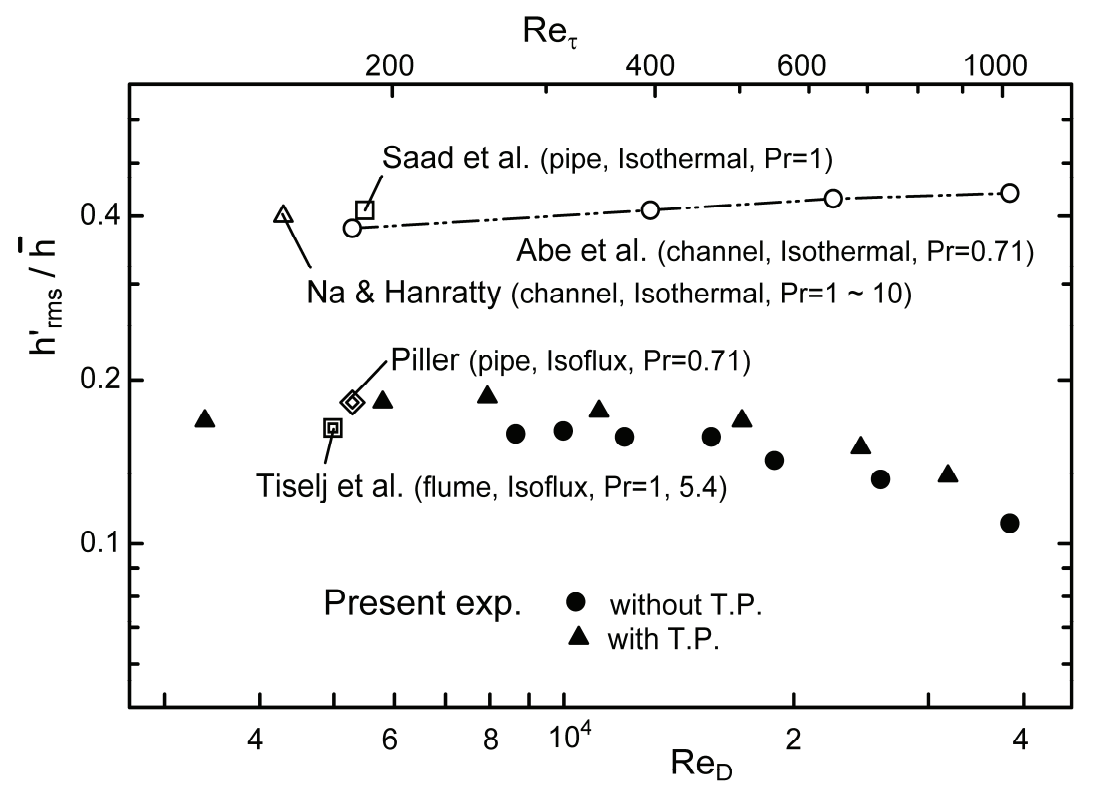

Fig. $8 \mathrm{Rms}$ value of the fluctuating heat transfer coefficient with respect to the Reynolds number, obtained herein compared with that estimated from the results of DNSs. The present value of $h_{r m s}^{\prime} / \bar{h}$ is approximately constant at 0.16 to 0.18 for the Reynolds number of $3,000<R e_{D}<12,000$ and appears reasonable compared with that of DNSs, which are under isoflux condition (experimental condition of the present study is similar to the isoflux condition).

observed in the present study, although the Prandtl number is different.

Moreover, the instantaneous temperature distribution can be compared to the flow field of the DNS performed by $\mathrm{Wu}$ and Moin (2008). In their study, the contours of the instantaneous streamwise velocity, $u_{z}$, at the near-wall region (at $0.01 R$ from the wall) were illustrated for the cases of $R e_{D}=5,300$ and 44,000 . At $R e_{D}=5,300$, the streaky structure of the contours is very similar to the thermal streaks obtained in the present study at a similar Reynolds number. The streaky structure was very fine at $R e_{D}=44,000$, which agrees with the trend of the present results for the higher Reynolds number.

As shown in Fig. 6, there exists a complicated spatio-temporal fluctuation in the heat transfer for the turbulent pipe flow, which causes problems related to wall temperature fluctuation. As such, the statistical values of the temporal fluctuation are investigated from the spatio-temporal distribution of the heat transfer coefficient obtained herein.

Figure 7 shows the temporal fluctuation of the local heat transfer coefficient at $R e_{D}=10,100$ corresponding to Fig. 6 at $z / D=10.8$ and $\theta=0^{\circ}$. The time trace of $h(t)$ (Fig. 7) is characterized by several maximum spikes, which are formed by the convection of the high heat-transfer spots in Fig. 6(a). Figure 8 shows the rms value of the fluctuating heat transfer coefficient at $z / D=10.8(z=220 \mathrm{~mm})$. Since the restoration rate of the heat transfer fluctuation decreases with increasing frequency, as described in Section 3.2, the evaluated heat transfer fluctuation $h_{r m s}^{\prime} / \bar{h}$ at $R e_{D} \geq 15,000$ decreases gradually with increasing Reynolds number. Nevertheless, the value of $h_{r m s}^{\prime} / \bar{h}$ is approximately constant at 0.16 to 0.18 for the lower Reynolds number of $3,000<R e_{D}<12,000$, at which the attenuation was considered to be small.

For comparison, the values of $h^{\prime}{ }_{r m s} / \bar{h}$ estimated from the results of DNSs performed by Na and Hanratty (2000), Tiselj et al. (2001), Abe et al. (2004), Piller (2005), and Saad et al. (2007) are plotted in Fig. 8, which shows that the value of $h_{r m s}^{\prime} / \bar{h}$ greatly depends on the difference in the thermal boundary condition, namely, $h_{r m s}^{\prime} / \bar{h} \approx 0.4$ for the isothermal condition (which corresponds to an infinite-heat-capacity wall), whereas $h_{r m s}^{\prime} / \bar{h}=0.16$ to 0.18 for the isoflux condition (which corresponds to a zero-heat-capacity wall). Since the experimental condition of the present study is similar to the isoflux condition, the value of $h_{r m s}^{\prime} / \bar{h}=0.16$ to 0.18 for the present experiment appears reasonable.

Based on the above results, it was demonstrated that quantitative measurement, not only of the time-averaged heat transfer, but also of the statistics of the temporal fluctuation, was possible using this technique. 


\section{Experiment - Heat transfer around an orifice}

Then, we used the technique using IRT to investigate the separated and reattached flow behind an orifice, where heat transfer fluctuates violently.

\subsection{Velocity distribution}

The velocity distribution across the pipe around the orifice plate (bore ratio: $d / D=0.49$ ) was measured beforehand using a laser Doppler velocimeter (LDV: Flow Explorer $75 \mathrm{~mW}$, DANTEC). The measurement was performed at $z / D=$ $-1,2.5$, and 9 (here, $z$ is the streamwise distance from the upstream edge of the orifice plate).

Figure 9 shows the streamwise velocity distributions across the pipe. Upstream of the orifice plate, at $z / D=-1$ (Fig. 9(a)), the velocity distribution is approximately the same as that for the fully developed pipe flow. In other words, the velocity distribution at $R e_{D}=10,000$ approximately follows the logarithmic law for a turbulent pipe flow. In contrast, downstream of the orifice, the separated flow at the front edge of the orifice plate reattaches at $z / D \approx 2.5$ in the case of a bore ratio of 0.5 , according to Deotte et al. (1991). In this region of $z / D=2.5$ (Fig. 9(b)), the mean velocity of the pipe center is remarkably high due to the flow contraction by the orifice. Moreover, the fluctuating velocity is very high, probably due to the flow reattachment caused by the flow instability in the separated shear layer. At $z / D=9$ (Fig. $9(\mathrm{c})$ ), the velocity distribution approaches that of a fully developed flow, but the velocity at the pipe center is shown to be flat, which is dissimilar to the logarithmic law.

\subsection{Mechanical vibration of thin heated surface}

Since the test surface, fabricated from $20.7-\mu \mathrm{m}$-thick titanium foil coated with black paint, was very thin, we examined the mechanical vibration of the surface due to the turbulent water flow. The vibration was measured using a laser displacement sensor (LK-G150, Keyence) in the flow reattachment region $\left(z / D=2.5, \theta=0^{\circ}\right)$, at which the

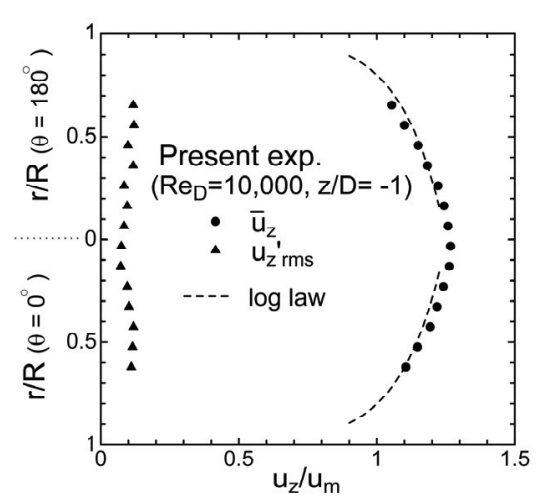

(a) At $z / D=-1\left(R e_{D}=10,000\right)$

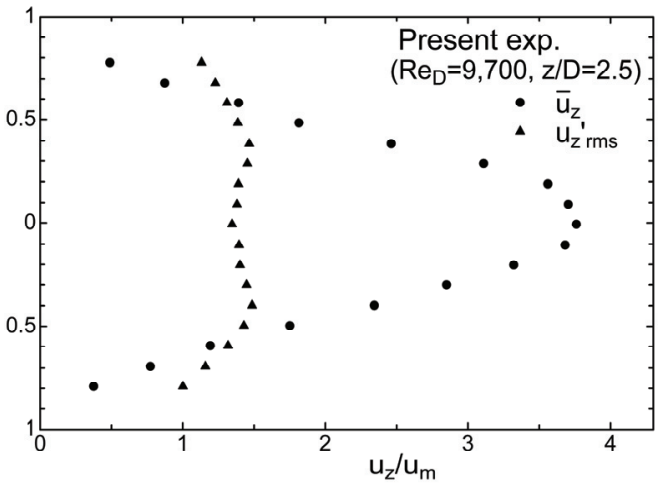

(b) At $z / D=2.5\left(R e_{D}=9,700\right)$

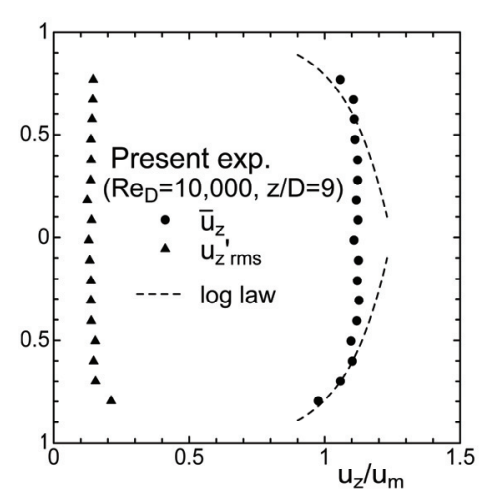

(c) At $z / D=9\left(R e_{D}=10,000\right)$

Fig. 9 Streamwise velocity distributions measured using LDV.

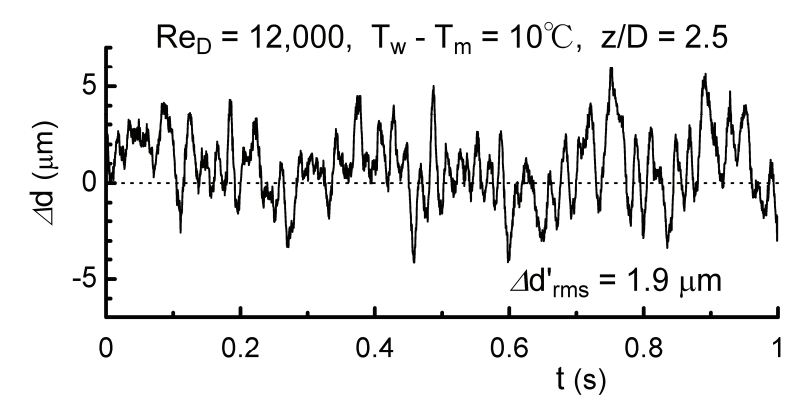

(a) On a thin test surface

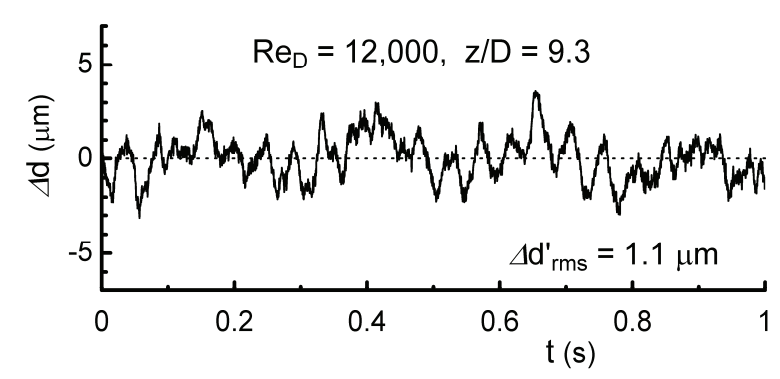

(b) On an acrylic pipe

Fig. 10 Mechanical vibration of the test model surface, measured using a laser displacement sensor 
vibration amplitude was considered to be large. Moreover, the vibration of the acrylic pipe $\left(z / D=9.3, \theta=0^{\circ}\right)$ was measured for comparison. Figure 10 shows the measurement results at $R e_{D}=12,000$. Although the high-frequency component of several-tens hertz for the test surface was amplified, the rms value of the displacement $\Delta d^{\prime}{ }_{r m s}$ was approximately $2 \mu \mathrm{m}$, which was not much larger than that for the acrylic pipe. Therefore, it is reasonable to assume that the test surface had sufficient rigidity against the turbulent water flow for the present experiment.

\subsection{Measurement of temperature distribution}

Figure 11 shows an instantaneous thermo-image for the entire region of the heated surface, as shown in Fig. 2, at $R e_{D}=12,100$. Although the detailed structure of the heat transfer cannot be discerned because of the lower spatial resolution, the qualitative behavior can be observed from this figure. The temperature of the heated surface immediately decreases downstream of the orifice plate due to the acceleration of the flow resulting from the contraction, and the flow reattachment. For $z / D>4$, the temperature gradually increases and approaches that at the upstream side of the orifice plate.

Figure 12 shows the instantaneous thermo-images taken in close proximity to the heated surface at $\operatorname{Re}_{D} \approx 12,000$. Upstream of the orifice plate at $z / D<-0.5$ (Fig. 12(a)), the temperature distribution is characterized by a streaky structure that was elongated in the streamwise direction. This structure flows downstream with some meandering,

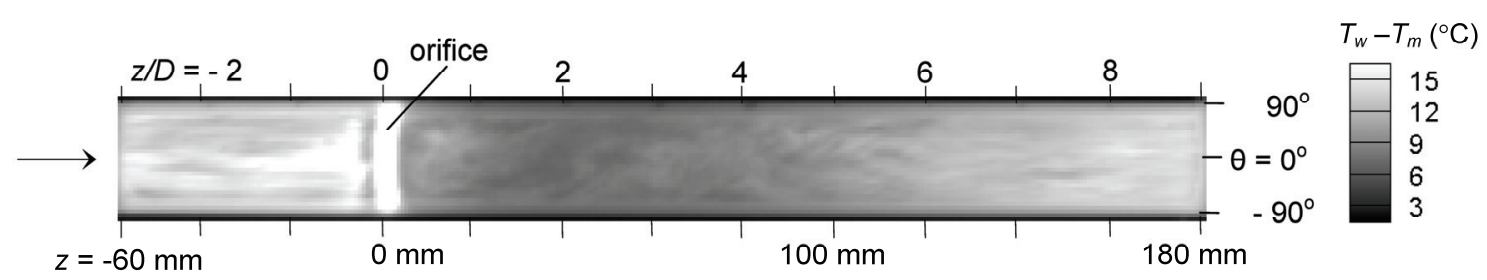

Fig. 11 Instantaneous thermo-image of the heated surface using IRT (wide measurement at $\operatorname{Re}_{D}=12,100$ ).

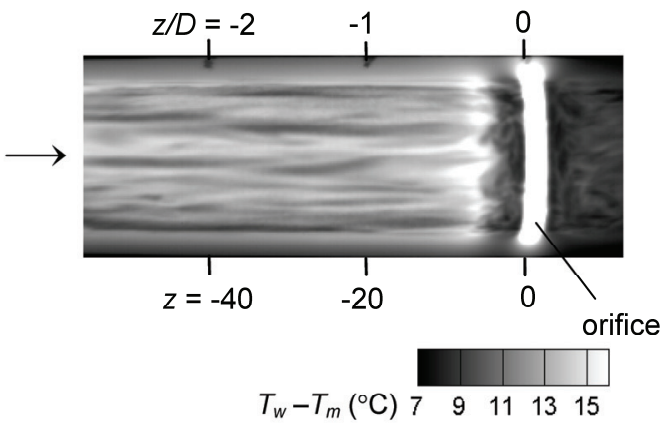

(a) $z / D=-2.7$ to $0.5(z=-55$ to $10 \mathrm{~mm})$

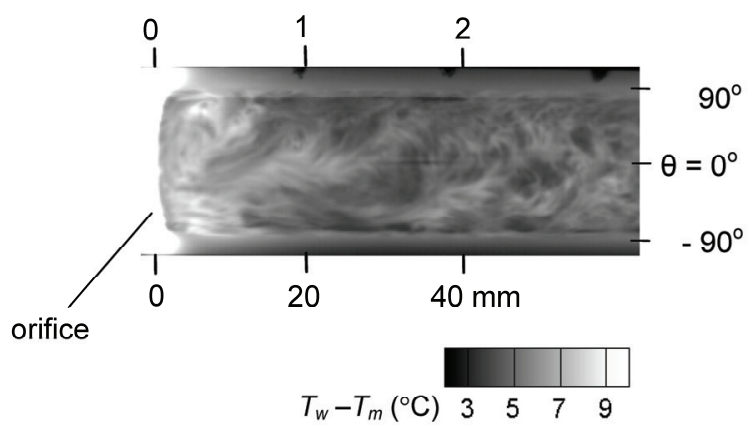

(b) $z / D=0$ to $3(z=0-60 \mathrm{~mm})$

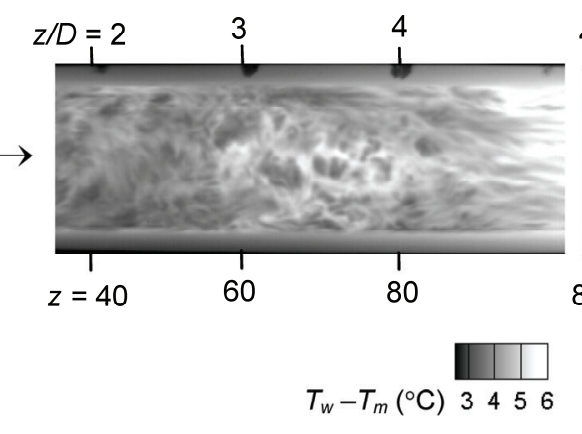

(c) $z / D=1.7$ to $5(z=35-100 \mathrm{~mm})$
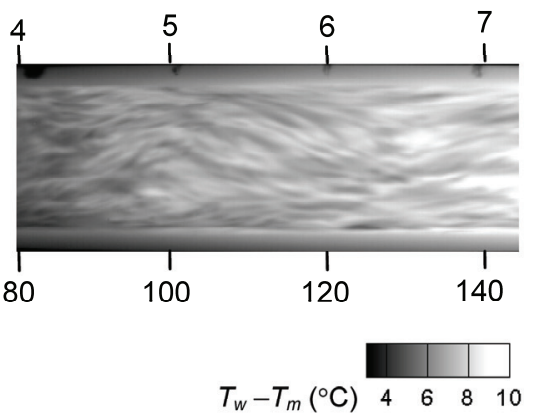
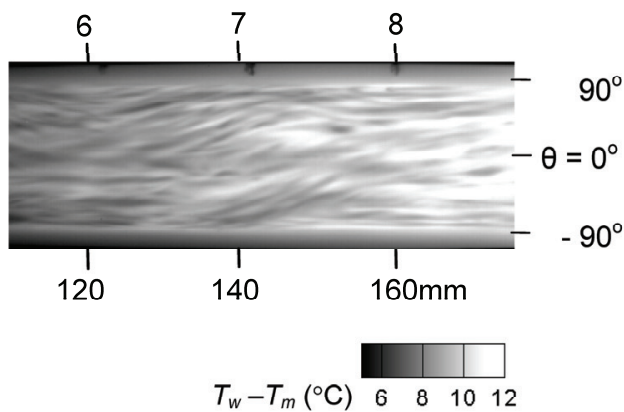

Fig. 12 Instantaneous thermo-images of the heated surface measured using IRT (close-up measurements at $R e_{D} \approx$

12,000). 
similar to that of the fully developed region in the pipe flow, as shown in Fig. 3(b). This indicates that the heat transfer at $z / D<-0.5$ was only slightly affected by the orifice plate. In contrast, the temperature immediately decreases just upstream of the orifice plate $(z / D>-0.25)$ probably due to the formation of the turbulent separation bubbles.

According to Deotte, et al. (1991), the separated flow at the orifice plate reattaches at $z / D \approx 2.5$ in the case of $d / D=$ 0.5 . Thus, for the present measurement, the flow reattachment was considered to occur at $z / D \approx 2.5$. Around this region (Figs. 12 (b) and 12 (c); $z / D \approx 2.5$ ), the temperature becomes very low and reveals very complicated structure, which is characterized by low-temperature spots that fluctuate violently in time and space. As shown in Figs. 12(b) and 12(c), these structures appear over $z / D=1$ to 4 , indicating that the heat transfer enhancement due to the flow reattachment appears to occur over a wide region.

At $z / D>4$, the low-temperature spots gradually change to have a streaky structure downstream. In the most downstream region of the present experiment $(z / D \approx 8)$, the streaky structure is still finer and meanders greatly compared to that upstream of the orifice plate. This indicates that the turbulent vortices at $z / D \approx 8$ have not yet recovered to those for the fully developed flow.

\subsection{Spatio-temporal heat transfer}

Figures 13 and 14 show the spatio-temporal heat transfer distribution at $R e_{D} \approx 12,000$, which was calculated in the same manner as that described in Section 3.2. In these figures, (a) is the instantaneous distribution, (b) is the time trace of the circumferential distribution, and (c) is the time trace of the streamwise distribution at $\theta=0^{\circ}$. In the instantaneous distribution of Fig. 13(a), the streaky structure elongates in the streamwise direction, similar to that for the fully developed flow (Fig. 6).

Downstream of the orifice (Fig. 14), the value of the heat transfer coefficient is several times higher than that upstream of the orifice, as indicated by the difference in the value of the grayscale. This heat transfer enhancement is considered to be due to the flow acceleration resulting from the contraction and the flow reattachment accompanied by the highly nonuniform and fluctuating flow. In the region over $z / D=1$ to 2.6 , the structure of the heat transfer enhancement becomes finer compared to that of the streaky structures as shown in Fig. 14(a). Although it cannot be confirmed from Fig. 14(a), finer structure of the heat transfer was observed in the region over $z / D=1$ to 4 . Moreover, as shown in Fig. 14(b), the duration of the heat transfer enhancement becomes very short (less than $20 \mathrm{~ms}$ ), and the peak value of the heat transfer enhancement becomes very high $\left(h \approx 24,000 \mathrm{~W} / \mathrm{m}^{2} \mathrm{~K}\right)$. Thus, the characteristic fluctuating frequency and its amplitude become very high in the reattachment region. At $z / D<2$, most of the high-heat-transfer spots move upstream with time, as shown in Fig. 14(c). This indicates that the backward flow is dominant in this region. In contrast, at $z / D=2$ to 2.5 , the high heat transfer spot moves backward and forward irregularly with time, as shown in Fig. 14(c). In this region, the heat transfer is much enhanced.

\subsection{Time-averaged and rms values of heat transfer}

Figures 15 shows local Nusselt number distribution along the streamwise direction at $\operatorname{Re}_{D} \approx 12,000(d / D=0.49, P r$ $=5$ ). The solid square symbols ( $\mathbf{a})$ indicate time-averaged values and the triangle symbols $(\boldsymbol{\Delta})$ indicate the rms values of the fluctuation. The vertical axis is normalized by the value in the fully developed region, $N u_{\infty}$, which is calculated by the empirical correlation of Gnielinski (1976). Upstream of the orifice plate $(z / D<-0.5)$, the Nusselt number is somewhat higher than that of the fully developed region (indicated by the horizontal dotted line) because the thermal field is not fully developed here. Just upstream of the orifice plate $(z / D \approx-0.25)$, the Nusselt number first exhibits a minimum and then increases immediately toward the orifice plate $(z / D \approx 0)$. This unique feature is considered to be reflected by the formation of turbulent separation bubbles. In this region, the rms value of the fluctuation also increases.

Downstream of the orifice plate $(z / D>0)$, the Nusselt number increases rapidly toward a maximum at $z / D \approx 2$, at which the value reaches four to five times higher than that for the fully developed pipe flow, as shown in Fig. 15. This maximum position of the Nusselt number does not coincide with the flow reattachment position of $z / D \approx 2.5$ reported by Deotte et al. (1991). There may be two possible reasons for this: 1) the flow reattachment position might not coincide with the maximum position of the heat transfer as reported by Suzuki et al. (1982), and 2) the flow field of the present experiment might be different from that of Deotte et al. (1991). In the future, we intend to discuss about the relationship between the heat transfer and the flow field.

At $z / D \approx 2$, the rms value of the fluctuation of the Nusselt number is over five times higher than that for the fully developed pipe flow, as shown in Fig. 15. This is considered to be due to the extremely complicated heat transfer 
structure (Fig. 14(a)). Downstream of this region, the time-averaged value and the rms value of the fluctuation gradually decrease and approach the fully developed value.

For comparison, the results of previous experiments by Krall and Sparrow $\left(1966\right.$, bore ratio $d / D=0.5, R_{D}=$

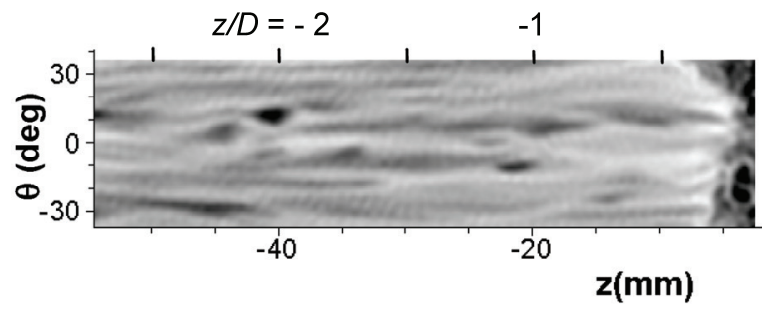

(a) Instantaneous distribution

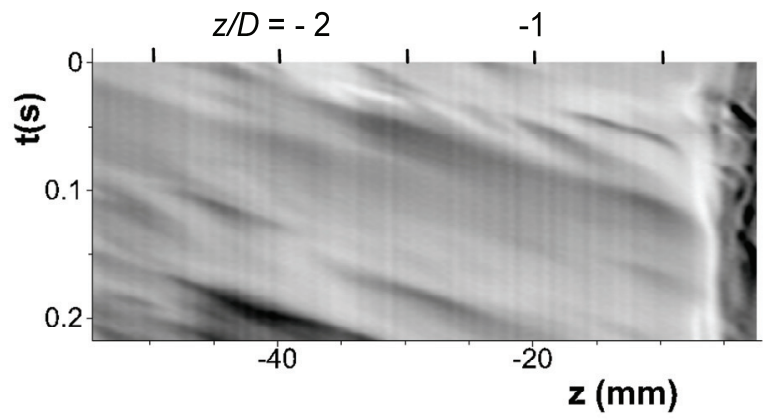

(c) Time trace along $z\left(\theta=0^{\circ}\right)$

Fig. 13 Spatio-temporal distribution of $h$ around an orifice at $z / D=-2.6$ to -0.1 , which is calculated by Eq. (4) $\left(R e_{D}=12,500, k_{\text {cut }}=1,190 \mathrm{~m}^{-1}, f_{\text {cut }}=200 \mathrm{~Hz}\right)$.

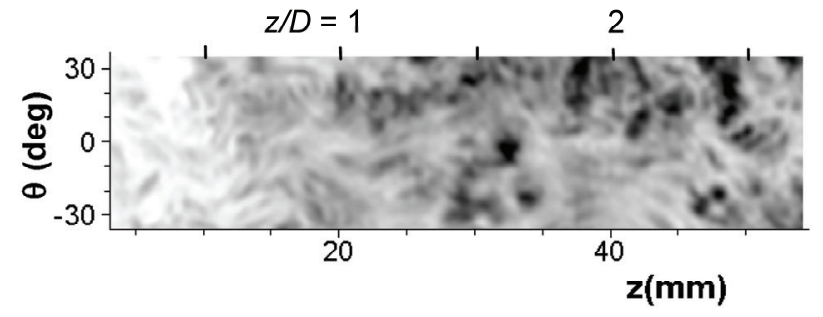

(a) Instantaneous distribution

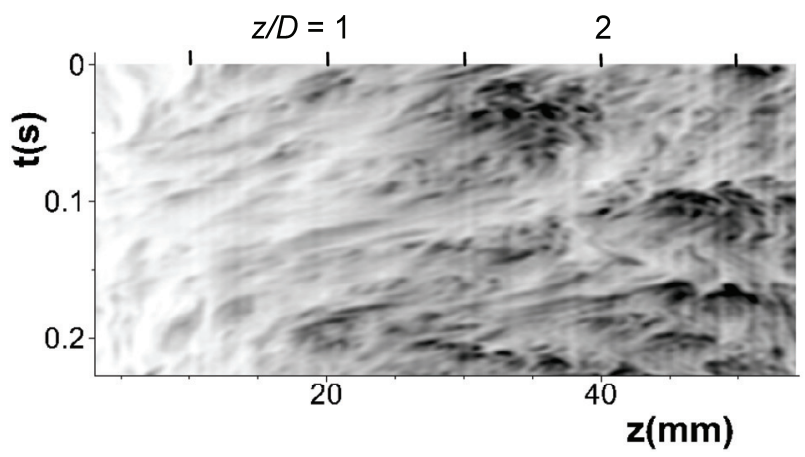

(c) Time trace along $z\left(\theta=0^{\circ}\right)$

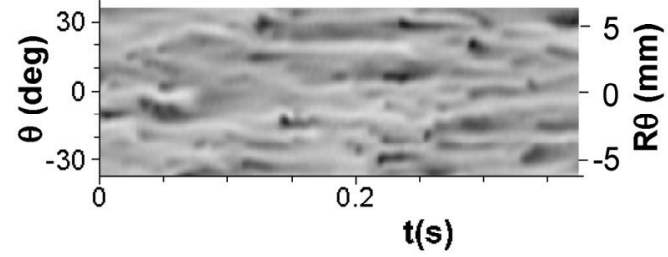

(b) Time trace along $\theta(z / D=-1)$
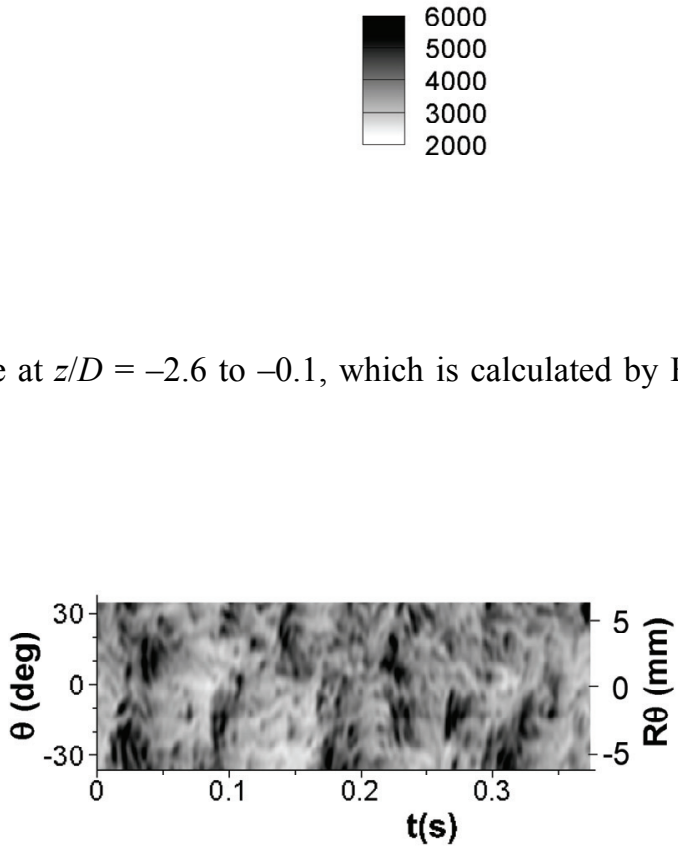

(b) Time trace along $\theta(z / D=2)$

Fig. 14 Spatio-temporal distribution of $h$ around an orifice at $z / D=0.2$ to 2.6, which is calculated by Eq. (4) (Re $e_{D}$ $=12,400, k_{\text {cut }}=1,190 \mathrm{~m}^{-1}, f_{\text {cut }}=200 \mathrm{~Hz}$ ). 


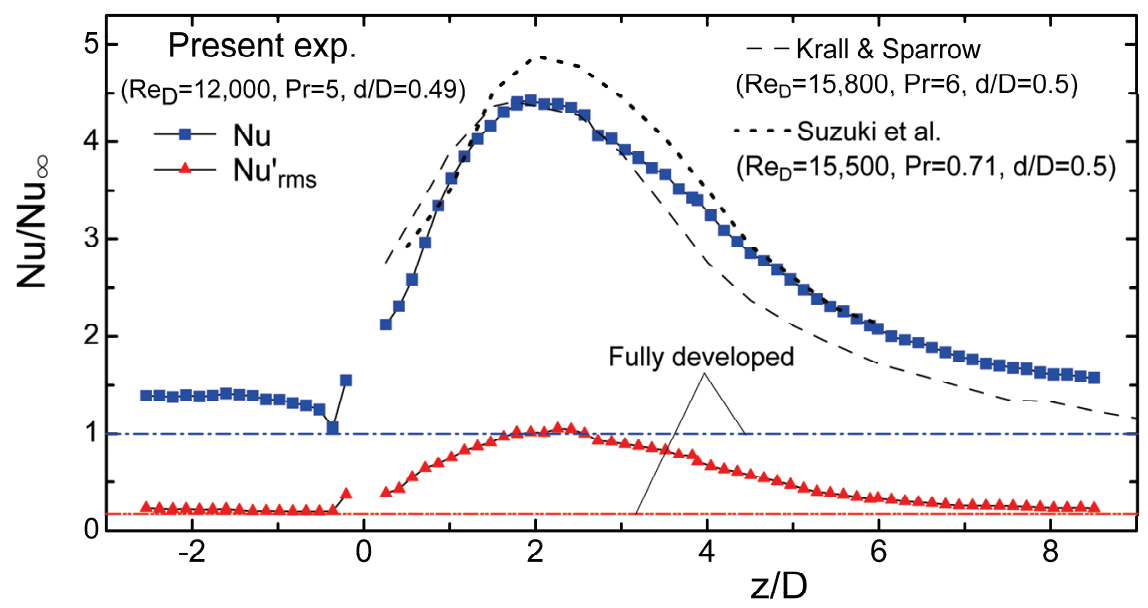

Fig. 15 Distribution of the time-averaged value and the rms value of Nusselt number fluctuation at $R e_{D} \approx$ 12,000 , obtained herein compared with the time-averaged value of previous experiments, which are conducted under similar experimental condition to the present study.

15,800, $\operatorname{Pr}=6$ ) and Suzuki et al. (1982, bore ratio $\left.d / D=0.5, R_{D}=15500, \operatorname{Pr}=0.71\right)$ are plotted in Fig. 15 . Here, the Nusselt numbers were normalized by the empirical correlation of Gnielinski (1976). The distribution of the Nusselt number agrees fairly well with those of previous experiments: the Nusselt number has a maximum at $z / D \approx 2$, the value of which is close to that of the present experiment. Moreover, the trend of the increase at $z / D<2$ and the decrease at $z / D>2$ is similar to that of the present experiment. Thus, it seems reasonable to conclude that the quantitative measurement is possible using this technique for the separated and reattaching flow, for which the heat transfer fluctuates in a very complicated manner in time and space.

\subsection{Spectral analysis of heat transfer fluctuation}

Spectral analysis was performed in order to investigate the characteristics of the heat transfer fluctuation. Figure 16 shows the pre-multiplied spectrum (PMS) of the local heat transfer fluctuation at $R e_{D} \approx 12,000$. The PMS is defined as follows:

$$
\operatorname{PMS}(f)=\frac{f \cdot P_{h}(f)}{\int_{-\infty}^{\infty} f \cdot P_{h}(f) \cdot d \log (f)} h_{r m s}^{\prime}{ }^{2}
$$

The value of the PMS plotted against the logarithm of the frequency shows the contribution to the variance of the heat transfer fluctuation. Upstream of the orifice (Fig. 16(a), $z / D=-1$ ), the fluctuation is dominant for a broad frequency band of 1 to $30 \mathrm{~Hz}$. In contrast, at the maximum position of the time-averaged heat transfer (Fig. 16(b), z/D = 2), the fluctuation intensity (the value of PMS) becomes much higher than that upstream of the orifice. At the same time, the frequency band increases to 3 to $100 \mathrm{~Hz}$, which is approximately three times higher than that upstream of the orifice. This is caused by the very complicated and finer structure of the heat transfer, as shown in Fig. 14(a). The heat transfer fluctuation and its frequency gradually decrease downstream (Fig. 16(b), $z / D=4$, Fig. 16(c), $z / D=8$ ). As mentioned above, it is revealed that the frequency of the heat transfer fluctuation around the flow reattachment region downstream of the orifice becomes remarkably high due to the very complicated and finer structure of the heat transfer. 


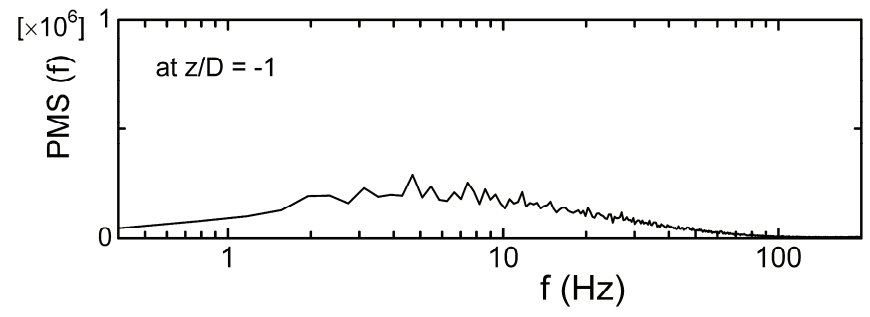

(a) $z / D=-1$

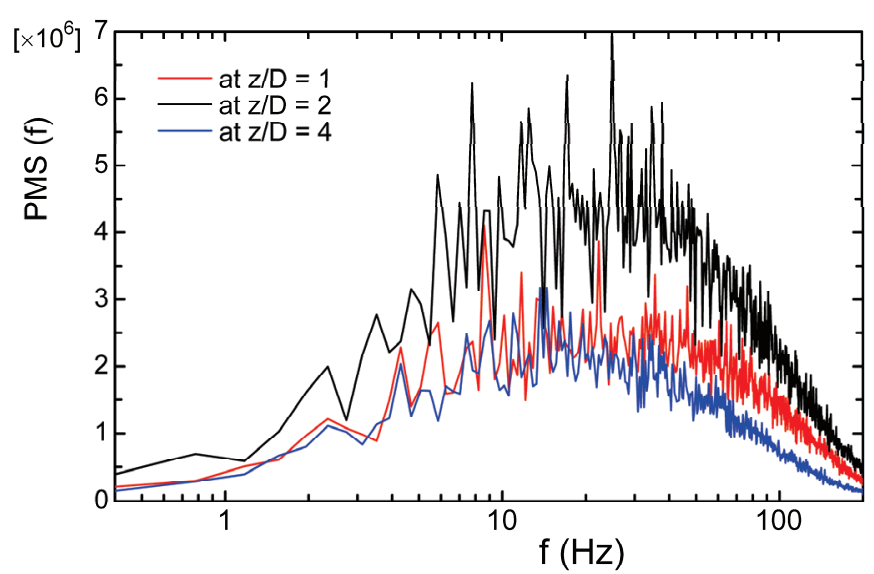

(b) $z / D=1,2,4$

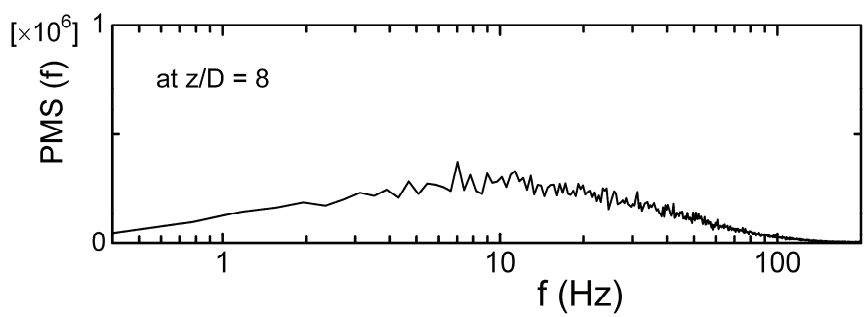

(c) $z / D=8$

Fig. 16 Pre-Multiplied Spectrum of heat transfer fluctuation at $R e_{D} \approx 12,000$. The value of the PMS plotted against the logarithm of the frequency shows the contribution to the variance of the heat transfer fluctuation. At the maximum position of the time-averaged heat transfer $(z / D=2)$, the fluctuation intensity (the value of PMS) becomes much higher than that upstream of the orifice. At the same time, the frequency-band shifts approximately three times higher than that upstream of the orifice.

\section{Conclusions}

A technique for measuring the spatio-temporal fluctuation of the heat transfer using high-speed infrared thermography was used to measure the heat transfer to a turbulent water pipe flow. The measurement was first performed for a fully developed pipe flow, and the results indicated that quantitative measurement was possible not only for time-averaged values, but also for fluctuating values.

The technique was then used to measure the heat transfer to the flow around an orifice (bore ratio of $0.49, R e_{D} \approx$ 12,000). The heat transfer increased remarkably downstream of the orifice. In the region over $z / D=1$ to 4 , the instantaneous structure of the heat transfer was remarkably finer than that for the streaky structure for the fully developed pipe flow. Moreover, the instantaneous structure of the heat transfer flows toward upstream and downstream irregularly at around $z / D=2$ to 2.5 , resulting in extremely complicated heat transfer fluctuation. Under the present experimental condition, the time-averaged heat transfer had a maximum at approximately two diameters downstream of the orifice, at which the value was four to five times higher than that for the fully developed pipe flow. In this region, the rms value of the fluctuation was over five times higher and its characteristic frequency-band shifted approximately 
three times higher than those for the fully developed pipe flow.

\section{Acknowledgments}

The authors wish to acknowledge Mr. Ryou Kitajima, Mr. Taishi Mizusawa, and Mr. Yusuf Wiranata Muhammad, former students of the National Defense Academy of Japan, for their assistance in conducting the experiments and data analysis.

\section{References}

Abe, H., Kawamura, H. and Matsu, Y., Surface heat-flux fluctuations in a turbulent channel flow up to $R e_{\tau}=1020$ with $\operatorname{Pr}=0.025$ and 0.71, International Journal of Heat and Fluid Flow, Vol.25 (2004), pp.404-419.

Deotte, R. E., Morrison, G. L., Panak, D. L. and Nail, G. H., 3-D laser doppler anemometry measurements of the axisymmetric flow field near an orifice plate, Flow Measurement and Instrumentation, Vol.2 (1991), pp.115-123.

Fujii, T., Fujii, M., and Honda, T., Theoretical and experimental studies of the free convection around a long horizontal thin wire in air, Proceedings of the 7th International Heat Transfer Conference (IHTC-7) (1982), pp.311-316.

Gnielinski, V., New equations for heat and mass transfer in turbulent pipe and channel flow, International Chemical Engineering, Vol.16, No.2 (1976), pp.359-367.

Hetsroni, G. and Rozenblit, R., Heat transfer to a liquid-solid mixture in a flume, International Journal of Multiphase Flow Vol.20, No.4 (1994), pp. 671-689.

Kawamura, T., Tanaka, S., Kumada, M. and Mabuchi, I., Time and spatial unsteady characteristics of heat transfer at the reattachment region of a backward-facing step, Transactions of the Japan Society of Mechanical Engineers, Series B, Vol.54, No.504 (1988), pp.2114-2120 (in Japanese).

Kawamura, T., Yamamori, M., Mimatsu, J. and Kumada, M., Three-dimensional unsteady characteristics of heat transfer around reattachment region of a backward-facing step flow, Transactions of the Japan Society of Mechanical Engineers, Series B, Vol.60, No.576 (1994), pp.2833-2839 (in Japanese).

Kimura, N. Ogawa, H. and Kamide, H., Experimental study on fluid mixing phenomena in T-pipe junction with upstream elbow, Nuclear Engineering and Design, Vol. 240 (2010), pp. 3055-3066.

Krall, K. M. and Sparrow, E. M., Turbulent heat transfer in the separated reattached, and redevelopment regions of a circular tube, Journal of Heat Transfer (1966), pp.131-136.

Mathie, R. and Markides, C.N., Heat transfer augmentation in unsteady conjugate thermal systems - Part I: semi-analytical 1-D framework, International Journal of Heat and Mass Transfer, Vol.56 (2013), pp.802-818.

Miyoshi, K., Nakamura, A., Utanohara, Y. and Takenaka, N., An investigation of wall temperature characteristics to evaluate thermal fatigue at a T-junction pipe, Mechanical Engineering Journal, Vol.1, No.5 (2014), pp.1-12.

Na, Y. and Hanratty, T. J., Limiting behavior of turbulent scalar transport close to a wall, International Journal of Heat and Mass Transfer, Vol.43 (2000), pp.1749-1758.

Nakagawa, S., Senda, M., Hiraide, A. and Kikkawa, S., Heat transfer characteristics in a channel flow with rectangular cylinder, Transactions of the Japan Society of Mechanical Engineers, Series B, Vol.64, No.622 (1998), pp.1817-1824 (in Japanese).

Nakamura, H., Measurements of time-space distribution of convective heat transfer to air using a thin conductive film, Transactions of the Japan Society of Mechanical Engineers, Series B, Vol.73, No.733 (2007), pp.1906-1914 (in Japanese).

Nakamura, H. and Yamada, S., Quantitative evaluation of spatio-temporal heat transfer to a turbulent air flow using a heated thin-foil, International Journal of Heat and Mass Transfer, Vol.64 (2013), pp.892-902.

Oyakawa, K., Miyagi, T., Oshiro, S., Senaha, I., Yaga, M. and Hiwada, M., Study on time-spatial characteristics of heat transfer by visualization of infrared and dye flow, Proceedings of 9th International Symposium on Flow Visualization (2000), p.233.

Petukhov, B.S., Heat transfer and friction in turbulent pipe flow with variable physical properties, Advances in Heat Transfer, Vol.6 (1970), pp.503-564.

Piller, M., Direct numerical simulation of turbulent forced convection in a pipe, International Journal for Numerical Methods in Fluids, Vol.49 (2005), pp.583-602. 
Saad, L.R., Rouiss, M.O. and Lauriat, G., Direct numerical simulation of turbulent heat transfer in pipe flows: Effect of Prandtl number", Int. J. Heat and Fluid Flow, Vol.28 (2007), pp.847-861.

Scholten, J. W. and Murray, D. B., Unsteady heat transfer and velocity of a cylinder in cross flow - I. Low freestream turbulence, International Journal of Heat and Mass Transfer, Vol.41, No.10 (1998), pp.1139-1148.

Shiibara, N., Nakamura, H. and Yamada, S., Visualization of turbulent heat transfer to a water flow in a circular pipe using high-speed infrared thermography, Journal of Flow Visualization and Image Processing, Vol.20 (1-2) (2013), pp.65-81.

Sorin, A., Bouloc, F., Bourouga, B. and Anthoine, P., Experimental study of periodic heat transfer coefficient in the entrance zone of an exhaust pipe, International Journal of Thermal Sciences, Vol. 47 (2008), pp. 1665-1675.

Suzuki, K., Sato, T., Kang, Y. and Sugimoto, T., Circular tube turbulent heat transfer in the downstream of an orifice, Heat transfer Japanese research, Vol.11, No.1 (1982), pp.70-90.

Tanaka, M., Takita, H., Monji, H. and Ohshima, H., Investigation on fluid-structure thermal interaction related to eddy structure on branch jet in T-junction, Transactions of the Japan Society of Mechanical Engineers, Series B, Vo.79, No.808 (2013), pp.2607-2611 (in Japanese).

The Japan Society of Mechanical Engineers ed., JSME standard, Guideline for evaluation of high-cycle thermal fatigue of a pipe (2003), The Japan Society of Mechanical Engineers (in Japanese).

The Japan Society of Thermophysical Properties ed., Thermophysical properties handbook (2008), p.25, The Japan Society of Thermophysical Properties (in Japanese).

Tiselj, I., Pogrebnyak, E., Li, C., Mosyak, A., and Hetsroni, G., Effect of wall boundary condition on scalar transfer in a fully developed turbulent flume, Physics of Fluids, Vol.13, No.4 (2001), pp.1028-1039.

$\mathrm{Wu}, \mathrm{X}$. and Moin, P., A direct numerical simulation study on the mean velocity characteristics in turbulent pipe flow, Journal of Fluid Mechanics, Vol.608 (2008), pp.81-112. 